\title{
THE EVANS LEMMA OF DIFFERENTIAL GEOMETRY
}

\author{
Myron W. Evans \\ Alpha Foundation for Advanced Study \\ E-mail: emyrone@aol.com
}

Received 25 October 2003

A rigorous proof is given of the Evans lemma of general relativity and differential geometry. The lemma is the subsidiary proposition leading to the Evans wave equation and proves that the eigenvalues of the d'Alembertian operator, acting on any differential form, are scalar curvatures. The Evans wave equation shows that the eigenvalues of the d'Alembertian operator, acting on any differential form, are eigenvalues of the index-contracted canonical energy momentum tensor $T$ multiplied by the Einstein constant $k$. The lemma is a rigorous and general result in differential geometry, and the wave equation is a rigorous and general result for all radiated and matter fields in physics. The wave equation reduces to the main equations of physics in the appropriate limits, and unifies the four types of radiated fields thought to exist in nature: gravitational, electromagnetic, weak and strong.

Key words: Evans lemma, Evans wave equation, differential geometry, general relativity, unified field theory, generally covariant electrodynamics, Evans-Vigier field $\mathbf{B}^{(3)}$.

\section{INTRODUCTION}

The original theory of general relativity [1] used Riemann geometry to develop a generally covariant theory of gravitation, i.e., a theory which is objective in all frames of reference, so that the equations of gravitation take the same form in any frame of reference, and no frame is a preferred frame. It did not prove to be possible to unify this theory with that of the electromagnetic field for several reasons, prominent among which is the fact that the Maxwell-Heaviside theory is one of 
special relativity. The nineteenth century Maxwell Heaviside theory was the precursor to special relativity, and was extended from electrodynamics to other areas of physics by Einstein in 1905, after work by Michelson, Morley, Fitzgerald, Lorentz, Poincaré and others. It is not sufficient to replace the ordinary derivative by the covariant derivative in Maxwell-Heaviside theory, because in Riemann geometry the torsion tensor is not used, and there is no concept of orbital or intrinsic spin in Einstein's original general relativity.

In contemporary differential geometry, however [2], nonEuclidean spacetime is defined by two differential forms for any connection: the torsion and Riemann forms. The former is a vector-valued two-form and the latter a tensor-valued two-form, and the tetrad postulate of differential geometry [2-6] proves that the covariant derivative of the tetrad vanishes for all possible types of spacetime. Recently the tetrad postulate has been developed into the most general known equation of physics, the Evans wave equation [3-7], which can be used to describe and inter-relate all known radiated and matter fields, thus opening up many new areas of physics hitherto unknown.

In this paper the subsidiary proposition (a lemma of differential geometry) leading to the Evans wave equation is proven with differential geometry. The lemma states that scalar curvature $R$ always occurs in nature as eigenvalues of the d'Alembertian operator $\square$ acting on any differential form. The Evans wave equation states that the product $k T=-R$ always occurs in nature as eigenvalues generated by $\square$ acting on any differential form. Here $k$ is the Einstein constant and $T$ is the index contracted energy-momentum tensor. Both the lemma and the wave equation apply in general relativity without any restriction, and therefore unify the theory of radiated and matter fields. This is a major advance in physics because the electromagnetic and gravitational fields become parts of the same theory of differential geometry, and so there exist hitherto unknown inter-relations between fields which could be of fundamental importance in future applications.

If the differential form is the tetrad $q_{\mu}^{a}$, the lemma is

$$
\square q_{\mu}^{a}=R q_{\mu}^{a},
$$

and the wave equations reads

$$
(\square+k T) q_{\mu}^{a}=0 .
$$

In Sec. 2 a rigorous proof of the lemma is given from the tetrad postulate, and it is shown that the lemma always leads to the wave equation given that

$$
-R=k T
$$

which must be interpreted as an equation valid for all radiated and matter fields, as first inferred, but not proved, by Einstein [1]. Equation 
(3) is the result of the Evans field equation [3-7]

$$
(R+k T) q_{\mu}^{a}=0
$$

Usually Eq. (3) is given a restricted interpretation as the contracted form of the Einstein field equation for gravitation. The more general Evans field equation (4) reduces to the Einstein field equation $[1,2]$ and also gives field equations for the gauge-invariant electromagnetic field.

In Sec. 3 it is emphasised that both the lemma and the wave equation are valid for any differential form, thus giving an entire class of Evans wave equations valid for all radiated and matter fields.

\section{PROOF OF THE EVANS LEMMA}

The tetrad can be defined by the equation

$$
V^{a}=q_{\mu}^{a} V^{\mu}
$$

where $V^{a}$ is any contravariant vector in the orthonormal basis, indexed $a$, and $V^{\mu}$ is any contravariant vector in the base manifold indexed $\mu$. For example, $V$ can represent position vectors $x$, so the tetrad becomes

$$
x^{a}=q_{\mu}^{a} x^{\mu}
$$

and has sixteen independent components, the sixteen irreducible representations of the Einstein group [8-12]. The vector $V$ can also represent metric vectors $q$ [3-7], so the tetrad can be defined by

$$
q^{a}=q_{\mu}^{a} q^{\mu}
$$

This means that the symmetric metric [2-7] can be defined in general by the dot product of tetrads:

$$
q_{\mu \nu}^{(S)}=q_{\mu}^{a} q_{\nu}^{b} \eta_{a b}
$$

where $\eta_{a b}$ is the metric $\operatorname{diag}(-1,1,1,1)$ of the orthogonal space [2]. The antisymmetric metric is defined in general by the wedge product of tetrads:

$$
q_{\mu \nu}^{c(A)}=q_{\mu}^{a} \wedge q_{\nu}^{b}
$$

and the general metric tensor with sixteen independent components is defined by the outer product of tetrads:

$$
q_{\mu \nu}^{a b}=q_{\mu}^{a} q_{\nu}^{b}=q_{\mu}^{a} \otimes q_{\nu}^{b}
$$


The dot product is the gauge invariant gravitational field, the cross product the gauge invariant electromagnetic field, and the outer product combines the two fields and defines the way in which one influences the other.

The tetrad can also be defined [2] by basis vectors; for example,

$$
\hat{e}_{(\mu)}=q_{\mu}^{a} \hat{e}_{(a)}
$$

for covariant basis vectors, or

$$
\hat{\theta}^{(a)}=q_{\mu}^{a} \hat{\theta}^{(\mu)}
$$

for contravariant, or orthogonal, basis vectors. It can be seen that the tetrad always links quantities defined in the orthonormal space indexed $a$ to quantities defined in the base manifold, indexed $\mu$. As a simplified, less abstract, aid to understanding, the orthonormal space [2] can be thought of as the tangent space, and the basis vectors of the orthonormal space can be thought of as the Cartesian unit vectors in a system of axes defining a tangent to a curve in ordinary three dimensional space (the base manifold). The curve is defined in the base manifold, the tangent to the curve is defined in the tangent space. In general the orthonormal space can be [2] any abstract space whose coordinate system is always orthogonal (i.e., Euclidean) and whose unit vectors are normalized, as in gauge field theory. In general relativity, the orthonormal space is the tangent space to the base manifold. The development and experimental verification of $\mathrm{O}(3)$ electrodynamics [812] showed that the orthonormal space of gauge field theory can be thought of as a physical space, and this is the key to field unification with differential geometry. All indices $a$ in the new unified field theory [3-7] are indices of a physical space, tangent to a physical base manifold. The base manifold is four-dimensional non-Euclidean spacetime.

The tetrad can also be defined in terms of:

Pauli matrices,

$$
\hat{\sigma}_{(\mu)}=q_{\mu}^{a} \hat{\sigma}_{(a)},
$$

Pauli two-spinors,

$$
\phi^{a}=q_{\mu}^{a} \phi^{\mu}
$$

Dirac four-spinors,

$$
\psi^{a}=q_{\mu}^{a} \psi^{\mu}
$$

or Dirac matrices,

$$
\gamma^{a}=q_{\mu}^{a} \gamma^{\mu}
$$

The tetrad is also the generalization of the Lorentz transformation to general relativity:

$$
x^{a \prime}=q_{\mu}^{a \prime} x^{\mu} .
$$


This equation represents a generally covariant transformation [2]. The tetrad can also be a generally covariant transformation matrix between gauge fields:

$$
\psi^{a \prime}=q_{\mu}^{a \prime} \psi^{\mu} .
$$

So

$$
q_{\mu}^{a}=\frac{\partial V^{a}}{\partial V^{\mu}}, \frac{\partial q^{a}}{\partial q^{\mu}}, \frac{\partial x^{a}}{\partial x^{\mu}}, \ldots
$$

The tetrad is also the keystone to the representation and generalization of the Dirac equation in general relativity [15]. In terms of two-spinors, the generally covariant Dirac equation is

$$
\begin{aligned}
\phi_{R}^{a} & =\left(q_{\mu}^{a}\right)_{R} \phi_{L}^{\mu}(0), \\
\phi_{L}^{a} & =\left(q_{\mu}^{a}\right)_{L} \phi_{R}^{\mu}(0) .
\end{aligned}
$$

In terms of four-spinors, the same equation is

$$
\psi^{a}(p)=q_{\mu}^{a} \psi^{\mu}(0) .
$$

This illustrates some of the ways in which the tetrad can be used. In general it is an invertible matrix (2) with a well-defined inverse:

$$
q_{\mu}^{a} q_{a}^{\nu}=\delta_{\mu}^{\nu}
$$

where $\delta_{\mu}^{\nu}$ is the Kronecker delta function. The dimensionality of the tetrad matrix depends on the way in which it is defined; for example, using Eqs. (6),(7),(11), or (12), the tetrad is a $4 \times 4$ matrix; using Eq. (13), it is a $2 \times 2$ complex matrix. The name "tetrad" is used generically [2].

The tetrad is a vector-valued one-form, i.e., is a one form $q_{\mu}$ with labels $a$. If $a$ takes the values 1,2 , or 3 of a Cartesian representation of the tangent space, for example, the vector

$$
\mathbf{q}_{\mu}=q_{\mu}^{1} \mathbf{i}+q_{\mu}^{2} \mathbf{j}+q_{\mu}^{3} \mathbf{k}
$$

can be defined in this space. Each of the components $q_{\mu}^{1}, q_{\mu}^{2}$, or $q_{\mu}^{3}$ are scalar-valued one-forms of differential geometry [2], and each of $q_{\mu}^{1}, q_{\mu}^{2}$, and $q_{\mu}^{3}$ is therefore a covariant four-vector in the base manifold. The three scalar-valued one-forms are therefore the three components of the vector-valued one-form $q_{\mu}^{a}$, the tetrad form [2].

The power of differential geometry is that it inter-relates differential forms (and thus gives us new equations of geometry, topology and physics) without needing to know the details of the geometry of the base manifold. 
The well-known tetrad postulate [2-7] is

$$
D_{\mu} q_{\nu}^{a}=\partial_{\mu} q_{\nu}^{a}+\omega_{\mu b}^{a} q_{\nu}^{b}-\Gamma_{\mu \nu}^{\lambda} q_{\lambda}^{a}=0
$$

and is the basis of the Evans lemma and Evans wave equation [3-7] of differential geometry, the most powerful and general wave equation known in general relativity, and thus in physics. This statement is based on the fact that differential geometry is the most powerful and general form of non-Euclidean geometry. In order to help understanding of the lemma and wave equation, we first give the detailed proof of the tetrad postulate.

The proof inter-relates the definitions of the covariant derivative $D$ of a vector $X$ in the base manifold and tangent space and shows that the tetrad postulate is the direct consequence of these definitions. In the base manifold, the vector $X$ is defined by its components $X^{\nu}$ in the basis set $\partial_{\nu}$ and the covariant derivative $D$ by its components $D_{\mu}$ in the basis set $d x^{\mu}$. Thus

$$
D X=\left(D_{\mu} X^{\nu}\right) d x^{\mu} \otimes \partial_{\nu}=\left(\partial_{\mu} X^{\nu}+\Gamma_{\mu \lambda}^{\nu} X^{\lambda}\right) d x^{\mu} \otimes \partial_{\nu}
$$

where $\Gamma_{\mu \lambda}^{\nu}$ is the Chistoffel connection [2]. Similarly, in the tangent space,

$$
D X=\left(D_{\mu} X^{a}\right) d x^{\mu} \otimes \hat{e}_{(a)}=\left(\partial_{\mu} X^{a}+\omega_{\mu b}^{a} X^{b}\right) d x^{\mu} \otimes \hat{e}_{(a)},
$$

where $\omega_{\mu b}^{a}$ is the spin connection [2]. Using the following definitions:

$$
\begin{aligned}
& X^{a}=q_{\nu}^{a} X^{\nu}, \\
& \partial_{a}=q_{a}^{\sigma} \partial_{\sigma},
\end{aligned}
$$

Eq. (26) can be rewritten as

$$
D X=q_{a}^{\sigma}\left(q_{\nu}^{a} \partial_{\mu} X^{\nu}+X^{\nu} \partial_{\mu} q_{\nu}^{a}+\omega_{\mu b}^{a} q_{\lambda}^{b} X^{\lambda}\right) d x^{\mu} \otimes \partial_{\sigma},
$$

where the commutator rule for matrices has been used. Replacing the dummy (i.e., repeated) indices $\sigma$ by $\nu$ and using

$$
q_{a}^{\nu} q_{\nu}^{a}=1
$$

we obtain

$$
D X=\left(\partial_{\mu} X^{\nu}+q_{a}^{\nu} \partial_{\mu} q_{\lambda}^{a} X^{\lambda}+q_{a}^{\nu} q_{\lambda}^{b} \omega_{\mu b}^{a} X^{\lambda}\right) d x^{\mu} \otimes \partial_{\nu}
$$

Comparing Eqs. (25) and (31), one gets

$$
\Gamma_{\mu \lambda}^{\nu}=q_{a}^{\nu} \partial_{\mu} q_{\lambda}^{a}+q_{a}^{\nu} q_{\lambda}^{b} \omega_{\mu b}^{a} .
$$


Multiply both sides of this equation by $q_{\mu}^{a}$ to obtain the tetrad postulate

$$
q_{\nu}^{a} \Gamma_{\mu \lambda}^{\nu}=\partial_{\mu} q_{\lambda}^{a}+q_{\lambda}^{b} \omega_{\mu b}^{a} .
$$

It is seen that the postulate is valid [2] for any Christoffel or spin connection and for any base manifold. The importance of this is that the postulate is true for manifolds with non-zero torsion form as well as non-zero Riemann form. In the Evans unified field theory [3-7] the torsion form represents the gauge invariant electromagnetic field, and the Riemann form represents the gauge invariant gravitational field. The tetrad form represents the potential fields in both cases, and the torsion form is the Hodge dual of the Riemann form. These concepts are summarised in Table 1 of the Appendix.

In contrast, in Einstein's generally covariant theory of gravitation, nineteenth century Riemann geometry is still used and in this theory the torsion tensor is defined as zero by the metric compatibility condition [2] of Riemann geometry used to define the Christoffel connection in terms of the symmetric, ten component, metric tensor. Therefore electromagnetism cannot be represented by Riemannian geometry, and this is the reason why Einstein was not able to unify gravitation and electromagnetism. The absence of the torsion tensor is also the reason why electromagnetism cannot be made into a theory of Einsteinian general relativity simply by replacing the derivatives of the Maxwell Heaviside field equations by the covariant derivatives with Christoffel connection. This is a self-inconsistent procedure because it attempts to represent electromagnetism with spacetime curvature without defining spacetime torsion, or spin. When one self-consistently includes the torsion tensor, electromagnetism becomes a theory of general relativity defined essentially by the wedge product of tetrads

$$
T^{c}=R q^{a} \wedge q^{b}
$$

and by the Evans lemma and wave equation. The same theory [3-7] also describes gravitation, and the inter-relation between gravitation and electromagnetism. It can be seen from Eq. (34) that the torsion is a vector valued two form of differential geometry [2]. The fact that it is vector valued means that the internal index $c$ must have more than one component, and this means that electromagnetism cannot be a U(1) gauge field theory. The latter defines the electromagnetic potential with a scalar-valued one-form $A$, and the gauge-invariant electromagnetic field by the scalar valued two form $F$. Unfortunately the $\mathrm{U}(1)$ representation of electromagnetism is accepted as part of the standard model, but this is inconsistent with differential geometry (as well as with general relativity) for the above reasons. The first theory of electromagnetism that was found to be consistent with differential geometry was $\mathrm{O}(3)$ electrodynamics, which was based in turn on the Evans 
Vigier field $\mathbf{B}^{(3)}$ observed in the inverse Faraday effect [13]. The $\mathbf{B}^{(3)}$ field can now be recognized as the wedge product

$$
B^{(3)}=-i g A^{(1)} \wedge A^{(2)}
$$

in the complex circular basis [8-12]. The theory of $\mathrm{O}(3)$ electrodynamics is therefore the precursor of the Evans unified field theory based on the Evans lemma and wave equation, and $\mathrm{O}(3)$ electrodynamics has been extensively verified experimentally [8-12]. It can be made to take the form of two Maxwell Heaviside equations under certain well-defined conditions but it is a gauge field theory of fundamentally different symmetry, a theory in which the electromagnetic field is a torsion form as required, with well defined index $c$. The theory of $\mathrm{O}(3)$ electrodynamics is consistent with differential geometry because the torsion form is well defined, the index $c$ takes the values (1), (2), and (3) of the complex circular representation [8-12] of the tangent space. This is a physical representation of circular polarization, and is an illustration of the more general finding [3-7] that gauge theory can be unified with general relativity provided with the internal gauge space is a physical space.

In contrast, in the standard model, the internal space of a gauge field is abstract, and is the fiber bundle space, unrelated to the tangent space of general relativity. This leads to serious (and well known) internal inconsistencies in the standard model, in that only the gravitational field is generally covariant. The other three fields are manifestations of special relativity and an abstract gauge theory whose internal, or fiber bundle, index has no physical meaning of definition [2]. In the standard model the internal index of the gravitational field represents a physical tangent space, but the internal index of the other three fields represents an unphysical space. The standard model is also internally inconsistent, it does not define the torsion tensor as described already, i.e., the internal index for electromagnetism is missing.

The Evans lemma is a direct consequence of the tetrad postulate. The proof of the lemma starts from covariant differentiation of the postulate:

$$
D^{\mu}\left(\partial_{\mu} q_{\lambda}^{a}+\omega_{\mu b}^{a} q_{\lambda}^{b}-\Gamma_{\mu \lambda}^{\nu} q_{\nu}^{a}\right)=0
$$

Using the Leibnitz rule, we have

$$
\begin{gathered}
\left(D^{\mu} \partial_{\mu}\right) q_{\lambda}^{a}+\partial_{\mu}\left(D^{\mu} q_{\lambda}^{a}\right)+\left(D^{\mu} \omega_{\mu b}^{a}\right) q_{\lambda}^{b}+\omega_{\mu b}^{a}\left(D^{\mu} q_{\lambda}^{b}\right) \\
-\left(D^{\mu} \Gamma_{\mu \lambda}^{\nu}\right) q_{\nu}^{a}-\Gamma_{\mu \lambda}^{\nu}\left(D^{\mu} q_{\nu}^{a}\right)=0
\end{gathered}
$$

and so

$$
\left(D^{\mu} \partial_{\mu}\right) q_{\lambda}^{a}+\left(D^{\mu} \omega_{\mu b}^{a}\right) q_{\lambda}^{b}-\left(D^{\mu} \Gamma_{\mu \lambda}^{\nu}\right) q_{\nu}^{a}=0
$$

because

$$
D^{\mu} q_{\lambda}^{a}=D^{\mu} q_{\lambda}^{b}=D^{\mu} q_{\nu}^{a}=0
$$


The covariant divergence of a vector $V^{\mu}$ is defined [2] as

$$
D_{\mu} V^{\mu}=\partial_{\mu} V^{\mu}+\Gamma_{\mu \lambda}^{\mu} V^{\lambda}
$$

thus, using Eq. (40), it is found that

$$
D_{\mu} \partial^{\mu}=\partial_{\mu} \partial^{\mu}+\Gamma_{\mu \lambda}^{\mu} \partial^{\lambda}
$$

Rewriting the dummy indices inside the connection,

$$
D_{\mu} \partial^{\mu}=\partial_{\mu} \partial^{\mu}+\Gamma_{\nu \mu}^{\nu} \partial^{\mu}=\square+\Gamma_{\nu \mu}^{\nu} \partial^{\mu},
$$

and the wave equation (38) therefore becomes

$$
\left(\square+\Gamma_{\nu \mu}^{\nu} \partial^{\mu}\right) q_{\lambda}^{a}-R_{1} q_{\nu}^{a}=0,
$$

where the scalar curvature $R_{1}$ is defined by

$$
-R_{1} q_{\lambda}^{a}:=\left(D^{\mu} \omega_{\mu b}^{a}\right) q_{\lambda}^{b}-\left(D^{\mu} \Gamma_{\mu \lambda}^{\nu}\right) q_{\nu}^{a}
$$

Using the tetrad postulate,

$$
\partial_{\mu} q_{\lambda}^{a}=-\omega_{\mu b}^{a} q_{\lambda}^{b}+\Gamma_{\mu \lambda}^{\nu} q_{\nu}^{a}
$$

and thus

$$
\partial^{\mu} q_{\lambda}^{a}=-\omega_{b}^{\mu a} q_{\lambda}^{b}+\Gamma_{\lambda}^{\mu \nu} q_{\nu}^{a} .
$$

Therefore, in Eq. (43),

$$
\square q_{\lambda}^{a}-\Gamma_{\nu \mu}^{\nu} \omega_{b}^{\mu a} q_{\lambda}^{b}+\Gamma_{\nu \mu}^{\nu} \Gamma_{\lambda}^{\mu \nu} q_{\nu}^{a}-R_{1} q_{\lambda}^{a}=0 .
$$

Define the scalar curvature $R_{2}$ by

$$
-R_{2} q_{\lambda}^{a}:=-\Gamma_{\nu \mu}^{\nu} \omega_{b}^{\mu a} q_{\lambda}^{b}+\Gamma_{\nu \mu}^{\nu} \Gamma_{\lambda}^{\mu \nu} q_{\nu}^{a},
$$

to obtain the Evans lemma

$$
\square q_{\lambda}^{a}=R q_{\lambda}^{a}
$$

where

$$
R=R_{1}+R_{2} \text {. }
$$

Given the tetrad postulate, the lemma shows that scalar curvature $R$ is always an eigenvalue of the wave equation (49) for all spacetimes, in other words, $R$ is quantized. The eigenoperator is the d'Alembertian operator $\square$, and the eigenfunction in this case is the 
tetrad. In Sec. 3 we will show that the eigenfunction can be any differential form, thus introducing a powerful class of wave equations to differential geometry and physics. The lemma is the subsidiary proposition leading to the Evans wave Eq. (2) through Eq. (3). The lemma is an identity of differential geometry, and so is comparable in generality and power to the well known Poincaré lemma [14]. In other words, new theorems of topology can be developed from the Evans lemma in analogy with topological theorems $[2,14]$ from the Poincaré lemma. This can be the subject of future work in mathematics, work which may lead in turn to new findings in physics based on topology. The immediate importance of the lemma to physics is that it is the subsidiary proposition leading to the Evans wave equation, which is valid for all radiated and matter fields. Equation (49) can be solved for $R$ given tetrad components, or vice versa, solved for tetrad components for a given $R$. The equation is non-linear in the spin and Christoffel connections, but for a given $R$ it is a linear second order partial differential wave equation, or eigenequation. In this sense it is an equation of wave mechanics and therefore of quantum mechanics, and so unifies quantum mechanics, unified field theory and general relativity. Its power is therefore apparent and the wave equation (49) reduces to known equations of physics [3-7] in the appropriate limits. These include the four Newton equations, the Poisson equations of gravitation and electrostatics, the Schrödinger, Klein-Gordon and Dirac equations, and the equations of $\mathrm{O}(3)$ electrodynamics. Equation (49) produces the quark color triplet through a choice of eigenfunction (a three-spinor of the SU(3) representation), and so unifies the gravitational and strong fields. In the limit of special relativity [3-7],

$$
R \rightarrow m^{2} c^{2} / \hbar^{2}
$$

where $m$ is the particle mass, $\hbar$ Planck's constant, and $c$ the speed of light in vacuo. Equation (51) means that there exists a minimum mass density defined by a rest volume $V$ :

$$
\rho_{\min }=m / V=m^{2} c^{2} / \hbar^{2} k .
$$

The product $m V$ is a universal constant:

$$
m V=\hbar^{2} k / c^{2}
$$

Equation (52) has the important consequence that there must be a minimum amount of mass/energy in the universe, in other words the universe cannot be "empty" $(R=-k T=0)$. This minimum amount of energy is the zero point energy of the Evans wave equation of quantum physics, and sometimes this zero point energy is referred to as "vacuum energy." It is observed in the Casimir effect and in the Lamb shift for example. Electromagnetic energy can therefore be obtained 
from the vacuum [15], interpreted as spacetime with torsion, and such energy may have been observed in devices such as the motionless electromagnetic generator [16]. In the limit of classical special relativity this minimum amount of energy is the rest energy $m c^{2}$, and so the Evans wave equation gives a new meaning to the Einstein rest energy $m c^{2}-$ it is the special-relativistic limit of the scalar curvature $R$. In non-relativistic equations such as the Schrödinger equation the zero point energy (for example of a harmonic oscillator) does not have a classical meaning, but in relativistic equations such as the Evans equation it has a clear classical meaning, zero point energy originates in $R$. This is another important aspect of the Evans equation, it derives wellknown relativistic equations from differential geometry. The existence of a minimum density of mass/energy, defined by Eq. (52) also means that the volume $V$ is there at a maximum, the universe cannot have infinite extent, and can never, therefore be devoid entirely of matter or energy. The scalar curvature $R$ can therefore never be zero. In coming to these conclusions $T$ is recognized as the index contracted form of total energy/momentum, the sum of potential and kinetic terms. Total energy/momentum is always conserved through the Noether theorem $[2,14]$

$$
D_{\mu} T^{\mu \nu}=0
$$

\section{GAUGE INVARIANT FIELDS, DUALITY EQUA- TIONS, INHOMOGENEOUS FIELD EQUATIONS, AND THE CLASS OF EVANS EQUATIONS FOR ALL DIFFERENTIAL FORMS}

The wave equation (2) defines the potential fields [14] (Feynman's "universal influence") of gravitation and electromagnetism. The former is the tetrad $q_{\mu}^{a}$ and the latter is

$$
A_{\mu}^{a}=A^{(0)} q_{\mu}^{a}
$$

where $A^{(0)}$ is a $C$ negative coefficient [3-7]. The wave equation also shows how the metrics and gauge invariant fields of gravitation and electromagnetism emerge from the Evans field equation [3]:

$$
-R q_{\mu}^{a}=k T q_{\mu}^{a}
$$

Equation (56) can be expressed as $k T=-R$ and is the equation which transforms the lemma (49) into the wave equation (2). Using Eqs. (8) to (10), it can be seen that the Evans field equation (56) can be developed in at least three ways, to give

$$
-R q_{\mu \nu}^{(S)}=k T q_{\mu}^{a} q_{\nu}^{b} \eta_{a b}
$$




$$
\begin{gathered}
-R q_{\mu \nu}^{c(A)}=k T q_{\mu}^{a} \wedge q_{\nu}^{b}, \\
-R q_{\mu \nu}^{a b}=k T q_{\mu}^{a} \otimes q_{\nu}^{b},
\end{gathered}
$$

which are equations in the symmetric, antisymmetric and general metric tensors, respectively. Equation (57) has been shown [3-7] to the Einstein field equation, and Eq. (58) defines the gauge invariant electromagnetic field through the torsion tensor (34). Equation (59) defines the gauge invariant electromagnetic field through the torsion tensor (34). Equation (59) is an equation for the general metric, i.e., the general outer product of tetrads, and defines the way in which gravitation effects electromagnetism, and vice versa. A complete description of all radiated and matter fields requires the gauge invariant as well as the potential fields.

The gauge invariant gravitational field is defined [2] by the Riemann form of differential geometry through the second Maurer-Cartan structure relation

$$
R_{b}^{a}=D \wedge \omega_{b}^{a}
$$

The symbol $D \wedge$ denotes the covariant exterior derivative of differential geometry (see Table 1), (The spin connection $\omega_{b}^{a}$ does not transform, however, as a tensor [2] so $D \wedge$ is defined in this equation is an operator which is identical to, and has the same effect as, the covariant exterior derivative.) The gauge invariant electromagnetic field is defined by the torsion form of differential geometry through the first Maurer-Cartan structure relation:

$$
T^{a}=D \wedge q^{a},
$$

in which $q^{a}$ transforms as a tensor. The gauge invariant electromagnetic field is then

$$
G^{a}=D \wedge A^{a}=g A^{b} \wedge A^{c},
$$

where $G^{a}$ is a $C$ negative magnetic flux density (tesla $=$ weber $\mathrm{m}^{-2}$ ) and $1 / g$ is a fundamental or primordial magnetic flux (weber). The quantum (or minimum possible value) of magnetic flux is the universal constant $\hbar / e$ and is the magnetic fluxon [8-14]. This means that a minimum amount of magnetic flux is always present in the universe. In analogy a minimum amount of $T, m, R$, and $V$ is always present in the universe, as discussed already. The equivalent of Eqs. (61) and (62) in $\mathrm{U}(1)$ or Maxwell-Heaviside electrodynamics is

$$
F=d \wedge A,
$$

where $d \wedge$ is the exterior derivative (see Table 1 ). Comparing the two available definitions (34) and (61) of the electromagnetic field gives the cyclically symmetric equation of differential geometry

$$
D \wedge q^{a}=\kappa q^{b} \wedge q^{c}, \quad R=\kappa^{2},
$$


which generalizes the $B$ cyclic theorem of $\mathrm{O}(3)$ electrodynamics [8-12]. The cyclic equation (64) is generally covariant and gauge invariant. All the equations of the Evans unified field theory are generally covariant, in other words they are all equations of differential geometry. This means that in the limit of special relativity, they are all Lorentz covariant. Indeed, as we have seen in Eq. (17), the tetrad, the eigenfunction of the Evans lemma and wave equation, can be defined as the equivalent of Lorentz transform matrix in general relativity.

The homogeneous unified field equations of the Evans theory are the Bianchi identities (see Table 1)

$$
\begin{aligned}
& D \wedge R_{b}^{a}:=0, \\
& D \wedge T^{a}:=0 .
\end{aligned}
$$

In the Maxwell-Heaviside theory, the equivalent equation is

$$
d \wedge F:=0
$$

The Bianchi identities follow from the Poincaré lemma

$$
D \wedge D:=0,
$$

and Eq. (67) follows from the Poincaré lemma

$$
d \wedge d:=0
$$

The inhomogeneous field equations of the Evans theory are

$$
\begin{aligned}
& D \wedge{ }^{*} R_{b}^{a}=J_{b}^{a}, \\
& D \wedge{ }^{*} T^{c}=J^{c},
\end{aligned}
$$

where ${ }^{*} R_{b}^{a}$ is the dual of $R_{b}^{a}$, defined as in Table 1 , and where ${ }^{*} T^{c}$ is the dual of $T^{c}$. In Eq. (70), $J_{b}^{a}$ is a tensor-valued three-form, the current form of gravitation, and $J^{c}$ is a vector-valued three-form, the current form of electromagnetism. The equivalent of Eqs. (70) and (71) in the Maxwell-Heaviside field theory is

$$
d \wedge{ }^{*} F=J
$$

where ${ }^{*} F$ is the dual of $F$ and $J$ is a scalar-valued three-form, the current form of this theory $[2,14]$.

In the development of the Evans field theory, novel and important duality relations of differential geometry have been discovered (1) between the torsion and Riemann forms and (2) between the spin connection and tetrad. These are fundamental to differential geometry, to topology, and to generally covariant physics. They are proven from the fact that the tangent space is an orthonormal Euclidean space, so in 
this space there exists the relation [2-7] between any axial vector $V_{a}$ and its dual antisymmetric tensor $V_{\kappa}$ :

$$
V_{a}=\epsilon_{a b c} V_{b c},
$$

where $\epsilon_{a b c}$ is the Levi Civita symbol or three-dimensional totally antisymmetric unit tensor (see Table 1). Raising one index gives

$$
\epsilon_{b c}^{a}=\eta^{c d} \epsilon_{d b c}
$$

where $\eta^{a d}=\operatorname{diag}(-1,1,1,1)$ is the metric of the orthonormal space. The first Evans duality equation of differential geometry is

$$
R_{b}^{a}=\kappa \epsilon_{b c}^{a} T^{c}
$$

showing that the spin connection is dual to the Riemann form, and the second Evans duality equation of differential geometry is

$$
\omega_{b}^{a}=\kappa \epsilon_{b c}^{a} q^{c},
$$

showing that the spin connection is dual to the tetrad. The duality equations define the symmetry of the Riemann form because they imply

$$
R_{a b}=R \epsilon_{a b c d} q^{c} \wedge q^{d},
$$

where $\epsilon_{a b c d}$ is the four-dimensional totally antisymmetric unit tensor. Therefore the Riemann form is antisymmetric in its indices $a$ and $b$ and also antisymmetric in the indices $\mu$ and $\nu$ of the base manifold [2]. The symmetries of the Riemann form and Riemann tensor are explained in more detail in Table 1 . The duality relations greatly simplify the unified field theory and show that gravitation and electromagnetism are closely related by duality symmetry in differential geometry.

It has been shown that the tetrad can be defined and understood in a number of different ways (5) to (23), for example, and Table 1. From these and other relations it follows straightforwardly that the Evans lemma and wave equation apply to any differential form, so there exists a class of new wave equations in differential geometry, topology and general relativity. For example, the tetrad can be defined in terms of the metric vectors as in Eq. (7); and, using the Leibnitz theorem, it follows that

$$
(\square+k T) q^{\mu}=0 .
$$

Similarly,

$$
\begin{aligned}
& (\square+k T) T^{a}=0, \\
& (\square+k T) R_{b}^{a}=0,
\end{aligned}
$$

and so on.

Acknowledgments. Craddock Inc., the Ted Annis Foundation, and the Association of Distinguished American Scientists are thanked for funding this work, and AIAS colleagues for interesting discussions. 


\section{APPENDIX}

Table 1. Glossary of new results and fundamental definitions

\begin{tabular}{|c|c|c|}
\hline 1 & $D_{\mu} q_{\nu}^{a}=0$ & The tetrad postulate \\
\hline 2 & $D q^{a}=0$ & $\begin{array}{l}\text { The tetrad postulate in the } \\
\text { rotation of differential geometry }\end{array}$ \\
\hline 3 & $\begin{array}{l}d()=\partial_{\mu}() \\
D()=D_{\mu}() \\
d \wedge() \\
D \wedge()\end{array}$ & $\begin{array}{l}\text { Partial derivative } \\
\text { Covariant derivative } \\
\text { Exterior derivative } \\
\text { Exterior covariant derivative }\end{array}$ \\
\hline 4 & $D_{\mu} V^{\nu}=\partial_{\mu} V^{\nu}+\Gamma_{\mu \lambda}^{\nu} V^{\lambda}$ & $\begin{array}{l}\text { Covariant derivative of a } \\
\text { vector } V^{\nu}\end{array}$ \\
\hline 5 & $\begin{array}{l}(d X)_{\mu \nu}^{a}=\left(\partial \wedge X^{a}\right)_{\mu \nu} \\
=\left(d \wedge X^{a}\right)_{\mu \nu} \\
=\partial_{\mu} X_{\nu}^{a}-\partial_{\nu} X_{\mu}^{a}=\left[\partial_{\mu}, X_{\mu}^{a}\right]\end{array}$ & $\begin{array}{l}\text { The exterior derivative in } \\
\text { component notation }\end{array}$ \\
\hline 6 & $d \wedge X^{a}$ & $\begin{array}{l}\text { The exterior derivative in the } \\
\text { notation of differential geometry }\end{array}$ \\
\hline 7 & $\begin{array}{l}D \wedge X^{a} \\
=d \wedge X^{a}+\omega_{b}^{a} \wedge X^{b} \\
\left(D \wedge X^{a}\right)_{\mu \nu} \\
=\partial_{\mu} X_{\nu}^{a}-\partial_{\nu} X_{\mu}^{a} \\
\quad+\omega_{\mu b}^{a} X_{\nu}^{b}-\omega_{\nu b}^{a} X_{\mu}^{b}\end{array}$ & $\begin{array}{l}\text { The exterior covariant } \\
\text { derivative, where } \omega_{b}^{a} \text { is i.e., } \\
\text { the spin connection }\end{array}$ \\
\hline 8 & $\begin{array}{l}D_{\mu} q_{\lambda}^{a}=\partial_{\mu} q_{\lambda}^{a} \\
\quad+\omega_{\mu b}^{a} q_{\lambda}^{b}-\Gamma_{\mu \lambda}^{\nu} q_{\nu}^{a}=0\end{array}$ & $\begin{array}{l}\text { Covariant derivative of the } \\
\text { tetrad, denoted by } D q^{a}\end{array}$ \\
\hline 8 & $\partial_{\mu} \phi=D_{\mu} \phi$ & Definition when $\phi$ is a scalar \\
\hline 9 & If $\phi=0, D_{\mu} 0=\partial_{\mu} 0:=0$ & Identity of geometry \\
\hline 10 & $\begin{array}{l}\qquad d\left(D q^{a}\right):=0 \\
\text { i.e., } \partial_{\mu}\left(D^{\mu} q_{\nu}^{a}\right):=0\end{array}$ & $\begin{array}{l}\text { Wave equation of } \\
\text { differential geometry }\end{array}$ \\
\hline 11 & $D D=d D=\square-R$ & $\begin{array}{l}\text { The Evans lemma of } \\
\text { differential geometry }\end{array}$ \\
\hline 12 & $\square q^{a}=R q^{a}$ & $\begin{array}{l}\text { The Evans lemma as an } \\
\text { eigenequation }\end{array}$ \\
\hline 13 & $R=-k T$ & $\begin{array}{l}\text { Index contracted form of } \\
\text { the Einstein field equation }\end{array}$ \\
\hline
\end{tabular}




\begin{tabular}{|c|c|c|}
\hline 14 & $(\square+k T) q^{a}:=0$ & $\begin{array}{l}\text { The Evans wave equation } \\
\text { in the notation of differential } \\
\text { geometry }\end{array}$ \\
\hline 15 & $(\square+k T) q_{\mu}^{a}:=0$ & $\begin{array}{l}\text { The Evans wave equation in } \\
\text { component notation }\end{array}$ \\
\hline 16 & $R q^{a}=-k T q^{a}$ & $\begin{array}{l}\text { The Evans field equation in } \\
\text { differential geometry }\end{array}$ \\
\hline 17 & $A^{a}=A^{(0)} q^{a}$ & $\begin{array}{l}\text { The electromagnetic potential } \\
\text { field (a tetrad one-form) }\end{array}$ \\
\hline 18 & $(\square+k T) A^{a}:=0$ & $\begin{array}{l}\text { Evans wave equation for } \\
\text { electrodynamics }\end{array}$ \\
\hline 19 & $\begin{array}{l}(A \wedge B)_{\mu \nu} \\
\quad=A_{\mu} B_{\nu}-A_{\nu} B_{\mu}\end{array}$ & $\begin{array}{l}\text { The wedge product in } \\
\text { component notation }\end{array}$ \\
\hline 20 & $G^{c}=G^{(0)} q^{a} \wedge q^{b}$ & $\begin{array}{l}\text { The gauge invariant } e / m \\
\text { field }\end{array}$ \\
\hline 21 & $T^{c}=R q^{a} \wedge q^{b}$ & $\begin{array}{l}\text { The torsion form, where } R \\
\text { is the scalar curvature }\end{array}$ \\
\hline 22 & $R_{b}^{a}=\epsilon_{b c}^{a} T^{c}$ & $\begin{array}{l}\text { The gauge invariant gravitational } \\
\text { field and the second Evans duality } \\
\text { relation }\end{array}$ \\
\hline 23 & $\epsilon_{b c}^{a}=\eta^{a d} \epsilon_{d b c}$ & $\begin{array}{l}\text { Definition of the Levi-Civita symbol } \\
\varepsilon_{b c}^{a} \text { in the orthonormal space } \\
\text { of the tetrad }\end{array}$ \\
\hline 24 & $\eta^{a d}=\operatorname{diag}(1,-1,-1,-1)$ & $\begin{array}{l}\text { Metric in the orthonormal space } \\
\text { of the tetrad }\end{array}$ \\
\hline 25 & $\epsilon_{a b c}=\left[\begin{array}{cc}1 & \text { even } \\
-1 & \text { odd } \\
0 & \text { otherwise }\end{array}\right.$ & $\begin{array}{l}\text { Levi-Civita symbol, the rank three } \\
\text { totally antisymmetric unit tensor }\end{array}$ \\
\hline 26 & $\omega_{b}^{a}=\kappa \epsilon_{b c}^{a} q^{c}$ & $\begin{array}{l}\text { The first Evans duality equation, } \\
\text { the spin connection is the dual } \\
\text { of the tetrad }\end{array}$ \\
\hline 27 & $T^{a}=D \wedge q^{a}$ & $\begin{array}{l}\text { The first Maurer-Cartan } \\
\text { structure relation }\end{array}$ \\
\hline
\end{tabular}




\begin{tabular}{|c|c|c|}
\hline 28 & $R_{b}^{a}=D \wedge \omega_{b}^{a}$ & $\begin{array}{l}\text { The second Maurer-Cartan } \\
\text { structure relation }\end{array}$ \\
\hline 29 & $D \wedge T^{a}:=R_{b}^{a} \wedge q^{b}$ & The first Bianchi identity \\
\hline 30 & $D \wedge R_{b}^{a}:=0$ & The second Bianchi identity \\
\hline 31 & $D \wedge D=d \wedge d:=0$ & The Poincaré lemma \\
\hline 32 & $T^{a}=D \wedge q^{a} ; D \wedge T^{a}=R_{b}^{a} \wedge q^{b}$ & $\begin{array}{l}\text { The generally covariant } \\
\text { theory of electromagnetism; } \\
\text { gauge invariant }\end{array}$ \\
\hline 33 & $R_{b}^{a}=D \wedge \omega_{b}^{a} ; D \wedge R_{b}^{a}:=0$ & $\begin{array}{l}\text { The generally covariant theory } \\
\text { of gravitation; gauge invariant }\end{array}$ \\
\hline 34 & $F=d \wedge A ; d \wedge F:=0$ & Maxwell-Heaviside theory \\
\hline 35 & $D \wedge\left({ }^{*} T^{a}\right)=0$ & $\begin{array}{l}\text { First homogenous Evans field } \\
\text { equation }\end{array}$ \\
\hline 36 & $D \wedge\left({ }^{*} R_{b}^{a}\right)=0$ & $\begin{array}{l}\text { Second homogenous Evans } \\
\text { field equation }\end{array}$ \\
\hline 37 & $d \wedge\left({ }^{*} F\right)=J$ & $\begin{array}{l}\text { The inhomogenous Maxwell- } \\
\text { Heaviside field equation }\end{array}$ \\
\hline 38 & $\left({ }^{*} T^{a}\right)^{\mu \nu}=\frac{1}{2} \epsilon^{\mu \nu \rho \sigma}\left(T^{a}\right)_{\rho \sigma}$ & Definition of ${ }^{*} T^{a}$ \\
\hline 39 & $\left({ }^{*} R_{b}^{a}\right)^{\mu \nu}=\frac{1}{2} \epsilon^{\mu \nu \rho \sigma}\left(R_{b}^{a}\right)_{\rho \sigma}$ & Definition of ${ }^{*} R_{b}^{a}$ \\
\hline 40 & $\mathbf{E}=\left(\phi^{(0)} / c^{2}\right) \mathbf{g}$ & $\begin{array}{l}\text { Evans electrogravitic equation } \\
\text { in the weak-field limit }\end{array}$ \\
\hline 41 & $\begin{array}{l}e^{2}+m^{2}=1 \\
e^{2}-m^{2}=0 \\
e^{2}=m^{2}=1 / 2\end{array}$ & $\begin{array}{l}\text { The Evans symmetry } \\
\text { condition of unified field } \\
\text { theory (in reduced units) }\end{array}$ \\
\hline 42 & $\begin{array}{l}e^{2}+m^{2} \neq 1 \\
e^{2}-m^{2} \neq 0 \\
e^{2} \neq m^{2}\end{array}$ & $\begin{array}{l}\text { The Evans symmery-breaking } \\
\text { rule for hybrid effects of } \\
\text { gravitation on electro- } \\
\text { magnetion (in reduced units) }\end{array}$ \\
\hline 43 & $D \wedge q^{a}=\kappa q^{b} \wedge q^{c}$ & $\begin{array}{l}\text { The Evans cyclic equation of } \\
\text { differential geometry (reduced } \\
\text { units) }\end{array}$ \\
\hline 44 & $\mathbf{B}^{(1)} \times \mathbf{B}^{(2)}=i B^{(0)} \mathbf{B}^{(3) *}$ & $\begin{array}{l}\text { The Evans B cyclic theorem } \\
\text { of } O(3) \text { electrodynamics }\end{array}$ \\
\hline
\end{tabular}




\begin{tabular}{|c|c|c|}
\hline 45 & $q_{\mu \nu}^{(S)}=q_{\mu}^{a} q_{\nu}^{b} \eta_{a b}$ & $\begin{array}{l}\text { The symmetric diagonal metric } \\
\text { (gravitation) }\end{array}$ \\
\hline 46 & $q_{\mu \nu}^{c(A)}=q_{\mu}^{a} \wedge q_{\nu}^{b}$ & $\begin{array}{l}\text { The antisymmetric metric } \\
\text { (electromagnetism) }\end{array}$ \\
\hline 47 & $q_{\mu \nu}^{a b}=q_{\mu}^{a} q_{\nu}^{b}=q_{\mu}^{a} \otimes q_{\nu}^{b}$ & $\begin{array}{l}\text { The outer product, combined } \\
\text { gravitational and } e / m \text { field }\end{array}$ \\
\hline 48 & $q^{a}=q_{\mu}^{a} q^{\mu}$ & Definition of metric vectors $q^{a}$ and $q^{\mu}$ \\
\hline 49 & $x^{a}=q_{\mu}^{a} x^{\mu}$ & Definition of position vectors \\
\hline 50 & $q_{\mu}^{a}=\partial x^{a} / \partial x^{\mu}$ & $\begin{array}{l}\text { The sixteen independent components } \\
q_{\mu}^{a} \text { of the tetrad, i.e., the } 16 \text { irreps } \\
\text { of the Einstein group }\end{array}$ \\
\hline 51 & $\begin{aligned} q_{\mu}^{0}= & \partial x^{0} / \partial x^{\mu} \\
& \vdots \\
q_{\mu}^{3}= & \partial x^{3} / \partial x^{\mu}\end{aligned}$ & $\begin{array}{l}\text { The generally covariant metric } \\
\text { four-vectors }\end{array}$ \\
\hline 52 & $\begin{array}{l}q_{\mu}^{a}=\frac{\partial V^{a}}{\partial V^{\mu}}=\frac{\partial q^{a}}{\partial q^{\mu}}=\frac{\partial x^{a}}{\partial x^{\mu}} \\
=\ldots \ldots\end{array}$ & $\begin{array}{l}\text { Generally covariant definitions of the } \\
\text { tetrad in terms of vectors }\end{array}$ \\
\hline 53 & $\hat{e}_{(\mu)}=q_{\mu}^{a} \hat{e}_{(a)}$ & $\begin{array}{l}\text { Basis vector definition of the } \\
\text { tetrad for any basis set }\end{array}$ \\
\hline 54 & $q_{a}^{\mu} q_{\nu}^{a}=\delta_{\nu}^{\mu}$ & Invertible matrix rule for the tetrad \\
\hline 55 & $\hat{\theta}^{(a)}=q_{\mu}^{a} \hat{\theta}^{(\mu)}$ & $\begin{array}{l}\text { Orthogonal basis vector definition } \\
\text { of the tetrad }\end{array}$ \\
\hline 56 & $\hat{\sigma}_{(\mu)}=q_{\mu}^{a} \hat{\sigma}_{(a)}$ & $\begin{array}{l}\text { Pauli matrix definition of the tetrad in } \\
\text { Euclidean limit }\end{array}$ \\
\hline 57 & $\hat{q}_{(\mu)}=q_{\mu}^{a} \hat{q}_{(a)}$ & $\begin{array}{l}\text { Sachs's basis-vector definition of the } \\
\text { tetrad }\end{array}$ \\
\hline 58 & $\psi^{a}=q_{\mu}^{a} \psi^{\mu}$ & Dirac spinor definition of the tetrad \\
\hline 59 & $\gamma^{a}=q_{\mu}^{a} \psi^{\mu}$ & Dirac matrix definition of the tetrad \\
\hline 60 & $\Lambda_{\mu}^{a} \rightarrow q_{\mu}^{a}$ & $\begin{array}{l}\text { Tetrad as generalization of the } \\
\text { Lorentz transform matrix } \Lambda_{\mu}^{a}\end{array}$ \\
\hline 61 & $x^{a \prime}=q_{\mu}^{a \prime} x^{\mu}$ & $\begin{array}{l}\text { Tetrad as the generally covariant } \\
\text { coordinate transformation }\end{array}$ \\
\hline
\end{tabular}




\begin{tabular}{|c|c|c|}
\hline 62 & $\psi^{a \prime}=q_{\mu}^{a \prime} \psi^{\mu}$ & $\begin{array}{l}\text { Tetrad as the generally covariant } \\
\text { gauge transformation between field } \\
\text { vectors } \psi^{\mu} \text { and } \psi^{a \prime}\end{array}$ \\
\hline 63 & $\phi^{a}=q_{\mu}^{a} \phi^{\mu}$ & Tetrad defined by two spinors \\
\hline 64 & $\begin{array}{l}\phi_{R}^{a}=\left(q_{\mu}^{a}\right)_{R} \phi_{L}^{\mu}(0) \\
\phi_{L}^{a}=\left(q_{\mu}^{a}\right)_{L} \phi_{R}^{\mu}(0)\end{array}$ & $\begin{array}{l}\text { The generally covariant Dirac } \\
\text { equation in terms of } \\
\text { two-spinors }\end{array}$ \\
\hline 65 & $\psi^{a}(p)=q_{\mu}^{a} \psi^{\mu}(0)$ & $\begin{array}{l}\text { The generally covariant Dirac } \\
\text { equation as a tetrad definition }\end{array}$ \\
\hline 66 & $(\square+k T) \psi^{a}=0$ & $\begin{array}{l}\text { The Evans equation with spinor } \\
\text { eigenfunction, a generally } \\
\text { covariant Dirac equation }\end{array}$ \\
\hline 67 & $V=V^{\mu} \hat{e}_{(\mu)}$ & Vector \\
\hline 68 & $V^{\mu}$ & Vector components, contravariant \\
\hline 69 & $\hat{e}_{(\mu)}$ & Basis vectors \\
\hline 70 & $V^{*}=V_{\mu} \hat{\theta}^{(\mu)}$ & Vector in the dual vector space \\
\hline 71 & $V_{\mu}$ & Vector components, covariant \\
\hline 72 & $\hat{\theta}^{(\mu)}$ & Basis vectors of the dual space \\
\hline 73 & $\begin{aligned} \delta_{\mu}^{\nu} & =1, \nu=\mu \\
& =0, \nu \neq \mu\end{aligned}$ & Kronecker delta function \\
\hline 74 & $\begin{aligned} \hat{\theta}^{(\nu)} \hat{e}_{(\mu)} & =\delta_{\mu}^{\nu} \\
V^{\mu} V_{\nu} & =\delta_{\nu}^{\mu} \\
q^{\nu} q_{\mu} & =\delta_{\mu}^{\nu} \\
q_{a}^{\nu} q_{\mu}^{a} & =\delta_{\mu}^{\nu}\end{aligned}$ & Defining relations \\
\hline 75 & $g^{\mu \nu} g_{\nu \rho}=g_{\rho \nu} g^{\nu \mu}=\delta_{\rho}^{\mu}$ & $\begin{array}{l}\text { Definition of the inverse metric } \\
\text { tensor given in terms of the } \\
\text { metric tensor } g_{\nu \rho}\end{array}$ \\
\hline 77 & $g^{\mu \nu} g_{\mu \nu}=4$ & Trace of the product in $(75)$ \\
\hline 78 & $V_{\mu}$ & $\begin{array}{l}\text { Components of the scalar- } \\
\text { valued one-form } V \text {. }\end{array}$ \\
\hline 79 & $V_{\mu}=\left[V_{0} \ldots V_{n}\right]$ & $\begin{array}{l}\text { Row vector representation } \\
\text { of the one form }\end{array}$ \\
\hline
\end{tabular}




\begin{tabular}{|c|c|c|}
\hline 80 & $V^{\mu}=\left(\begin{array}{c}V \\
\vdots \\
V^{n}\end{array}\right)$ & $\begin{array}{l}\text { Column vector representative } \\
\text { of the contravariant vector }\end{array}$ \\
\hline 81 & $\begin{array}{l}V_{\mu} V^{\mu}=\left(V_{0} \ldots V_{n}\right)\left(\begin{array}{c}\vdots \\
V^{n}\end{array}\right) \\
\quad=V_{0} V^{0}+\cdots+V_{n} V^{n} \\
\quad=\mathbf{V} \cdot \mathbf{V}=g_{\mu \nu} V^{\mu} V^{\nu} \\
\quad=g_{\mu}^{a} V^{\mu} V_{a}=\left(g_{\mu}^{a} V_{a}\right) V^{\mu}\end{array}$ & $\begin{array}{l}\text { Inner or dot product of covariant } \\
\text { and contravariant vectors, where } \\
g_{\mu \nu} \text { is the metric tensor and } q_{\mu}^{a} \\
\text { is the tetrad }\end{array}$ \\
\hline 82 & $V_{\mu}^{a}$ & $\begin{array}{l}\text { Components of the vector-valued } \\
\text { one-form } V^{a}\end{array}$ \\
\hline 83 & $V_{\mu \nu}^{a}=-V_{\nu \mu}^{a}$ & $\begin{array}{l}\text { Components of the vector-valued } \\
\text { two-form }\end{array}$ \\
\hline 84 & $V_{b \mu \nu}^{a}=-V_{b \nu \mu}^{a}$ & $\begin{array}{l}\text { Components of the tensor-valued } \\
\text { two-form }\end{array}$ \\
\hline 85 & $\phi$ & $\begin{array}{l}\text { Scalar component of the } \\
\text { zero-form }\end{array}$ \\
\hline 86 & $\epsilon_{\mu \nu \rho \sigma}=\left\{\begin{array}{cc}1, & \text { even } \\
-1, & \text { odd } \\
0, & \text { otherwise }\end{array}\right.$ & $\begin{array}{l}\text { Totally antisymmetric tensor in } \\
4 \mathrm{D} \text {, the same in all spacetimes }\end{array}$ \\
\hline 87 & $d \phi=\left(\partial \phi / \partial x^{\mu}\right) \hat{\theta}^{\mu}$ & Exterior derivative of a scalar \\
\hline 88 & $(d \phi)_{\mu}=\partial_{\mu} \phi=D_{\mu} \phi$ & $\begin{array}{l}\text { Relation between exterior, } \\
\text { partial and covariant derivative } \\
\text { of a scalar } \phi\end{array}$ \\
\hline 89 & $*(U \wedge V)_{i}=\epsilon_{i}^{j k} U_{j} V_{k}$ & $\begin{array}{l}\text { Hodge dual definition of the } \\
\text { cross product in } 3 \text {-space }\end{array}$ \\
\hline 90 & $\begin{array}{l}d \wedge F:=0 \\
\partial_{[\mu} F_{\nu} \lambda_{]}:=0 \\
\partial_{\mu} F_{\nu \lambda}+\partial_{\lambda} F_{\mu \nu}+\partial_{\nu} F_{\lambda \mu}:=0 \\
\partial_{\mu}^{*} F^{\mu \nu}:=0 \\
{ }^{*} F^{\mu \nu}=\frac{1}{2} \epsilon^{\mu \nu \rho \sigma} F_{\rho \sigma}\end{array}$ & $\begin{array}{l}\text { Equivalent representation of } \\
\text { the Jacobi identity, or first } \\
\text { Bianchi identity, the } \\
\text { homogeneous field equation }\end{array}$ \\
\hline 91 & $\begin{array}{l}(d V)_{\mu_{1} \ldots \mu_{p+1}} \\
=(p+1) \partial_{\left[\mu_{1} V_{\mu_{2}} \ldots \mu_{p_{1}}\right]}\end{array}$ & $\begin{array}{l}\text { Exterior derivative of the } \\
\text { scalar-valued }(p+1) \text {-form. }\end{array}$ \\
\hline
\end{tabular}




\begin{tabular}{|c|c|c|}
\hline 92 & $\begin{aligned} T_{[\mu \nu \rho] \sigma} & =\frac{1}{6}\left(T_{\mu \nu \rho \sigma}-T_{\mu \rho \nu \sigma}\right. \\
& +T_{\rho \mu \nu \sigma}-T_{\nu \mu \rho \sigma} \\
& \left.+T_{\nu \rho \mu \sigma}-T_{\rho \nu \mu \sigma}\right)\end{aligned}$ & $\begin{array}{l}\text { Example of the general rule } \\
\text { for the antisymmetric tensor; } \\
\text { odd number of exchanges } \\
\text { give a minus sign }\end{array}$ \\
\hline 93 & $U^{\mu}=d x^{\mu} / d \tau$ & $\begin{array}{l}\text { Velocity four-vector in } \\
\text { special relativity }\end{array}$ \\
\hline 94 & $p^{\mu}=m U^{\mu}$ & Momentum four-vector \\
\hline 95 & $T^{\mu \nu}=(\rho+p) u^{\mu} u^{\nu}+p \eta^{\mu \nu}$ & Energy-momentum tensor \\
\hline 96 & $D_{\mu} V_{\nu}=\partial_{\mu} V_{\nu}-\Gamma_{\mu \nu}^{\lambda} V_{\lambda}$ & $\begin{array}{l}\text { Covariant derivative of a } \\
\text { one-form }\end{array}$ \\
\hline 97 & $T_{\mu \nu}^{\lambda}=\Gamma_{\mu \nu}^{\lambda}-\Gamma_{\nu \mu}^{\lambda}$ & Torsion tensor \\
\hline 98 & $R_{\sigma \mu \nu}^{\rho}=-R_{\sigma \nu \mu}^{\rho}$ & Riemann tensor \\
\hline 99 & $\begin{array}{l}{\left[D_{\mu}, D_{\nu}\right] V^{\rho}} \\
\quad=R_{\sigma \mu \nu}^{\rho} V^{\sigma}-T_{\mu \nu}^{\lambda} D_{\lambda} V^{\rho}\end{array}$ & $\begin{array}{l}\text { Commutator of covariant } \\
\text { derivatives }\end{array}$ \\
\hline 100 & $\begin{aligned} R_{\rho \sigma \mu \nu} & =g_{\rho \lambda} R_{\sigma \mu \nu}^{\lambda} \\
R_{\rho \sigma \mu \nu} & =-R_{\sigma \rho \mu \nu} \\
& =-R_{\rho \sigma \nu \mu} \\
& =R_{\mu \nu \rho \sigma}\end{aligned}$ & $\begin{array}{l}\text { Symmetries of the Riemann } \\
\text { tensor }\end{array}$ \\
\hline 101 & $\begin{array}{l}R_{\rho[\sigma \mu \nu]}:=0 \\
=R_{\rho \sigma \mu \nu}+R_{\rho \mu \nu \sigma}+R_{\rho \nu \sigma \mu}\end{array}$ & First Bianchi identity \\
\hline 102 & $\begin{array}{l}R_{a b}=g_{a c} R_{b}^{c} \\
g_{a c}=\eta_{a c} \\
=\operatorname{diag}(1,-1,-1,-1)\end{array}$ & $\begin{array}{l}\text { Relation between Riemann } \\
\text { forms of differential } \\
\text { geometry }\end{array}$ \\
\hline 103 & $\xi^{\mu}=\left(\begin{array}{l}\xi^{1} \\
\xi^{2}\end{array}\right)$ & $\begin{array}{l}\text { The spinor, a vector in } 2-\mathrm{D} \\
\text { rep. space (Barut, }(1.42) \text { ) }\end{array}$ \\
\hline 104 & $\begin{aligned} \xi_{\mu} & =C_{\mu \nu} \xi^{\nu} \\
& =\left[\xi_{1}, \xi_{2}\right]\end{aligned}$ & $\begin{array}{l}\text { The covariant spinor (Barut, } \\
\text { Eq. (1.48)). This is a } \\
\text { scalar-valued one-form }\end{array}$ \\
\hline 105 & $\xi^{\alpha} \eta_{\alpha}=\xi_{\alpha} \eta^{\alpha}$ & $\begin{array}{l}\text { Scalar invariant dot product } \\
\text { of two spinors (Barut, }\left(1.45^{\prime}\right) \text { ) }\end{array}$ \\
\hline 106 & $\psi_{\mu}=\left[\xi_{1}^{(R)}, \xi_{2}^{(R)}, \xi_{1}^{(L)}, \xi_{2}^{(L)}\right]$ & $\begin{array}{l}\text { Dirac four-spinor, a one form } \\
\text { of differential geometry }\end{array}$ \\
\hline
\end{tabular}




\begin{tabular}{|c|c|c|}
\hline 107 & $\begin{array}{l}X=\sigma_{\mu} x^{\mu} \\
X_{\alpha \dot{\alpha}}=\left(\sigma_{\mu} x^{\mu}\right)_{\alpha \dot{\alpha}}\end{array}$ & $\begin{array}{l}\text { Relation between spinors and } \\
\text { vectors. Both spinors and } \\
\text { vectors describe the same base } \\
\text { manifold, so we must be able to } \\
\text { interrelate them (Barut, p. } 30 \text { ) }\end{array}$ \\
\hline 108 & $\begin{aligned} \xi_{\mu} \xi^{\mu *} & =\left|\xi_{1}^{2}\right|+\left|\xi_{2}^{2}\right| \\
& =\xi_{1} \xi_{1}^{*}+\xi_{2} \xi_{2}^{*} \\
& =\xi_{1} \xi^{1 *}+\xi_{2} \xi^{2 *}\end{aligned}$ & $\begin{array}{l}\text { Dot product of two } 2 \text {-spinors, } \\
\text { a scalar invariant }\end{array}$ \\
\hline 109 & $x_{i} x^{i}=x_{1}^{2}+x_{2}^{2}+x_{3}^{2}$ & $\begin{array}{l}\text { Dot product of two } 3 \text {-vectors, } \\
\text { a scalar invariant }\end{array}$ \\
\hline 110 & $\begin{array}{l}x_{1}^{2}+x_{2}^{2}+x_{3}^{2}=\xi_{1} \xi_{1}^{*}+\xi_{2} \xi_{2}^{*} \\
\text { (three-dimensional space) }\end{array}$ & $\begin{array}{l}\text { Equating two scalar invariants } \\
\text { in the same base manifold }\end{array}$ \\
\hline 111 & $\begin{array}{l}\sigma_{0}=\left(\begin{array}{ll}1 & 0 \\
0 & 1\end{array}\right), \sigma_{1}=\left(\begin{array}{ll}0 & 1 \\
1 & 0\end{array}\right) \\
\sigma_{2}=\left(\begin{array}{cc}0 & -i \\
i & 0\end{array}\right), \sigma_{3}=\left(\begin{array}{cc}1 & 0 \\
0 & -1\end{array}\right)\end{array}$ & $\begin{array}{l}\text { The set of Pauli matrices of } \\
\text { the } \mathrm{SU}(2) \text { group }\end{array}$ \\
\hline 112 & $\begin{array}{c}\sigma_{0} \mathbf{i} \cdot \mathbf{i}=\sigma_{1} \sigma_{1}=1_{2} \\
\sigma_{0} \mathbf{j} \cdot \mathbf{j}=\sigma_{2} \sigma_{2}=1_{2} \\
\sigma_{0} \mathbf{k} \cdot \mathbf{k}=\sigma_{3} \sigma_{3}=1_{2} \\
1_{2}:=\left(\begin{array}{ll}1 & 0 \\
0 & 1\end{array}\right)\end{array}$ & $\begin{array}{l}\text { Another example of inter- } \\
\text { relating scalars in the } \mathrm{O}(3) \\
\text { and } \mathrm{SU}(2) \text { reps. of } 3 \text {-D space, } \\
\text { in this case, the scalar } \\
\text { components, } 1 \text {, of } \sigma_{0}\end{array}$ \\
\hline
\end{tabular}

\section{REFERENCES}

1. A. Einstein, The Meaning of Relativity (Princeton University Press, 1921).

2. S. M. Carroll, Lecture Notes on General Relativity (University of California, Santa Barbara, Graduate Course on arXiv:grqe/9712019 vl 3 Dec., 1997).

3. M. W. Evans, Found. Phys. Lett. 16, 367 (2003).

4. M. W. Evans, Found. Phys. Lett. 16, 507 (2003).

5. M. W. Evans, Found. Phys. Lett. 17, 25 (2004).

6. M. W. Evans, Found. Phys. Lett. 17, 149 (2004).

7. M. W. Evans, Found. Phys. Lett. 17, 301 (2004).

8. M. W. Evans, Physica B 182, 227, 237 (1992).

9. M. W. Evans, J.-P. Vigier, et al., The Enigmatic Photon (Kluwer Academic, Dordrecht, 1994 to 2002, hardback and softback, in five volumes). 
10. M. W. Evans and L. B. Crowell, Classical and Quantum Electrodynamics and the $\mathbf{B}^{(3)}$ Field (World Scientific, Singapore, 2001).

11. M. W. Evans, ed., Modern Nonlinear Optics, a special topical issue of I. Prigogine and S. A. Rice, series eds., Advances in Chemical Physics, Vols. 119(1) to 119(3) (Wiley Interscience, New York, 2001, second and e-book editions).

12. L. Felker, ed., The Evans Equations (World Scientific, Singapore, 2004, in preparation).

13. M. Tatarikis et al., "Measurements of the inverse Faraday effect in high intensity laser produced plasmas," CFL Annual Report 1998/1999, EPSRC Rutherford Appleton Laboratories, m.tatarikis@ic.ac.uk.

14. L. H. Ryder, Quantum Field Theory, 2nd edn. (Cambridge University Press, 1996).

15. P. W. Atkins, Molecular Quantum Mechanics, 2nd edn. (Oxford University Press, 1983).

16. T. E. Bearden, in Ref. (12), Vol. 119(2), and www. cheniere.org. 